\title{
Some Reduction Formulae Associated with Gauss and Fox-Wright Hypergeometric Functions
}

\author{
M.I. Qureshi ${ }^{1}$, Saima Jabee ${ }^{1, *}$, Sulakshana Bajaj ${ }^{2}$ \\ ${ }^{1}$ Department of Applied Sciences and Humanities, Faculty of Engineering and Technology, \\ Jamia Millia Islamia (A Central University), New Delhi-110025, India \\ ${ }^{2}$ Department of Mathematics, Govt. College Kota, Kota, Rajasthan-324001, India \\ *Corresponding author: saimajabee007@gmail.com
}

Received January 20, 2018; Revised March 06, 2018; Accepted June 22, 2018

\begin{abstract}
In this paper, we describe some reduction formulae for Gauss' hypergeometric function and Fox-Wright hypergeometric function associated with suitable convergence conditions using series rearrangement technique.
\end{abstract}

Keywords: Generalized hypergeometric function, Fox-Wright generalized hypergeometric function, Pfaff-Kummer's linear transformation

2010 Mathematics Subject Classification: 33C05, 33C20, 33C60, 33C99.

Cite This Article: M.I. Qureshi, Saima Jabee, and Sulakshana Bajaj, "Some Reduction Formulae Associated with Gauss and Fox-Wright Hypergeometric Functions." Turkish Journal of Analysis and Number Theory, vol. 6, no. 3 (2018): 103-106. doi: 10.12691/tjant-6-3-7.

\section{Introduction and Basic Notations}

In the present paper, we shall use the following standard notations:

$$
\begin{aligned}
& \mathrm{N}:=\{1,2,3, \ldots\}, \mathrm{N}_{0}:=\{0,1,2,3, \ldots\}=\mathrm{N} \cup\{0\} \\
& \mathrm{Z}_{0}^{-}:=\{0,-1,-2,-3, \ldots\},
\end{aligned}
$$$$
\mathbf{Z}^{-}:=\{-1,-2,-3, \ldots\}=\mathbf{Z}_{0}^{-} \backslash\{0\} \text { and } \mathbf{Z}=\left(\mathbf{Z}_{0}^{-} \cup \mathbf{Z}\right) \text {. }
$$

Here, as usual, $Z$ denotes the set of integers, $R$ denotes the set of real numbers, $\mathrm{R}^{+}$denotes the set of positive real numbers and $C$ denotes the set of complex numbers.

The Pochhammer symbol (or the shifted factorial) $(\lambda)_{v}$ $(\lambda, v \in \mathrm{C})$ is defined in terms of the familiar Gamma function by

$$
\begin{aligned}
& (\lambda)_{v}:=\frac{\Gamma(\lambda+v)}{\Gamma(\lambda)} \\
& = \begin{cases}\frac{(-1)^{n} k !}{(k-n) !} ; & \left(\lambda=-k ; v=n ; n, k \in \mathrm{N}_{0} ; 0 \leq n \leq k\right) \\
\lambda(\lambda+1) \ldots & (\lambda+n-1) ;\left(v=n \in \mathrm{N} ; \lambda \in \mathrm{C} \backslash \mathrm{Z}_{0}^{-}\right) \\
0 ; & \left(\lambda=-k ; v=n ; n, k \in \mathrm{N}_{0} ; n>k\right) \\
\frac{(-1)^{n}}{(1-\lambda)_{n}} ; & (v=-n ; n \in \mathrm{N} ; \lambda \neq 0, \pm 1, \pm 2, \ldots) .\end{cases}
\end{aligned}
$$

it being understood conventionally that $(0)_{0}=1$, and assumed tacitly that the Gamma quotient exists.

In the Gaussian hypergeometric series ${ }_{2} F_{1}(a, b ; c ; z)$, there are two numerator parameters $a, b$ and one denominator parameter $c$. A natural generalization of this series is accomplished by introducing any arbitrary number of numerator and denominator parameters. The non-terminating hypergeometric series [[5], pp.42-43]

$$
\begin{aligned}
& { }_{p} F_{q}\left[\begin{array}{l}
\alpha_{1}, \alpha_{2}, \ldots, \alpha_{p} ; \\
\beta_{1}, \beta_{2}, \ldots, \beta_{q} ;
\end{array}\right] \\
& =\sum_{n=0}^{\infty} \frac{\left(\alpha_{1}\right)_{n}\left(\alpha_{2}\right)_{n} \ldots\left(\alpha_{p}\right)_{n}}{\left(\beta_{1}\right)_{n}\left(\beta_{2}\right)_{n} \ldots\left(\beta_{q}\right)_{n}} \frac{z^{n}}{n !},
\end{aligned}
$$

is known as the generalized Gauss and Kummer series, or simply, generalized hypergeometric series. Here $p$ and $q$ are positive integers or zero (interpreting an empty product as unity), and we assume that the variable $z$, the numerator parameters $\alpha_{1}, \alpha_{2}, \ldots, \alpha_{p}$ and the denominator parameters $\beta_{1}, \beta_{2}, \ldots, \beta_{q}$ take on complex values, provided that

$$
\beta_{j} \neq 0,-1,-2, \ldots ; \quad j=1,2, \ldots, q .
$$

Convergence conditions [[5], p.43]:

Suppose that none of the numerator parameters is zero or negative integer (otherwise the question of convergence will not arise), and with the usual restriction (1.3), the ${ }_{p} F_{q}$ series in the definition (1.2)

(i) converges for $|z|<\infty$, if $p \leq q$,

(ii) converges for $|z|<1$, if $p=q+1$. 
Furthermore, if we denote

$$
\omega=\sum_{j=1}^{q} \beta_{j}-\sum_{i=1}^{p} \alpha_{i}
$$

it is known that the ${ }_{p} F_{q}$ series, with $p=q+1$, is

(a) absolutely convergent for $|z|=1$, if $\mathfrak{R}(\omega)>0$,

(b) conditionally convergent for $|z|=1, \quad z \neq 1$, if $-1<\Re(\omega) \leq 0$.

Fox-Wright generalized hypergeometric function of one variable:

The Fox-Wright $\Psi$ function of one variable ([[3], p.389]; see also $[4,6,7])$ is given by

$$
\begin{gathered}
p_{p} \Psi_{q}\left[\begin{array}{l}
\left(\alpha_{1}, A_{1}\right), \ldots,\left(\alpha_{p}, A_{p}\right) ; \\
\left(\beta_{1}, B_{1}\right), \ldots,\left(\beta_{q}, B_{q}\right) ;
\end{array}\right] \\
=\sum_{n=0}^{\infty} \frac{\Gamma\left(\alpha_{1}+A_{1} n\right) \ldots \Gamma\left(\alpha_{p}+A_{p} n\right)}{\Gamma\left(\beta_{1}+B_{1} n\right) \ldots \Gamma\left(\beta_{q}+B_{q} n\right)} \frac{z^{n}}{n !} \\
=\frac{\Gamma\left(\alpha_{1}\right) \ldots \Gamma\left(\alpha_{p}\right)}{\Gamma\left(\beta_{1}\right) \ldots \Gamma\left(\beta_{q}\right)} \sum_{n=0}^{\infty} \frac{\left(\alpha_{1}\right)_{n A_{1}} \ldots\left(\alpha_{p}\right)_{n A} p}{\left(\beta_{1}\right)_{n B_{1}} \ldots\left(\beta_{q}\right)_{n B_{q}}} \frac{z^{n}}{n !}, \\
=\frac{\Gamma\left(\alpha_{1}\right) \ldots \Gamma\left(\alpha_{p}\right)}{\Gamma\left(\beta_{1}\right) \ldots \Gamma\left(\beta_{q}\right)} \Psi_{q}^{*}\left[\begin{array}{l}
\left(\alpha_{1}, A_{1}\right), \ldots,\left(\alpha_{p}, A_{p}\right) ; \\
\left(\beta_{1}, B_{1}\right), \ldots,\left(\beta_{q}, B_{q}\right) ;
\end{array}\right], \\
=\frac{1}{2 \pi \rho} \int_{L} \frac{\prod_{i=1}^{q} \Gamma\left(\beta_{j}-B, \zeta\right)}{\prod_{j=1}^{p}\left(\alpha_{i}-A_{i} \zeta\right)}(-z)^{-\zeta} d \zeta,
\end{gathered}
$$

where $\rho^{2}=-1, z \in \mathrm{C}$; parameters $\alpha_{i}, \beta_{j} \in \mathrm{C}$; coefficients $A_{i}, B_{j} \in \mathrm{R}=(-\infty,+\infty)$ in case of series (1.4) (or $A_{i}, B_{j} \in \mathrm{R}^{+}(0,+\infty)$ in case of contour integral (1.6)), $A_{i} \neq 0(i=1,2, \ldots, p), \quad B_{j} \neq 0(j=1,2, \ldots, q)$. In equation (1.4), the parameters $\alpha_{i}, \beta_{j}$ and coefficients $A_{i}, B_{j}$ are adjusted in such a way that the product of Gamma functions in numerator and denominator should be well defined $[1,2]$.

$$
\begin{gathered}
\Delta^{*}=\left(\sum_{j=1}^{q} B_{j}-\sum_{i=1}^{p} A_{i}\right) \\
\delta^{*}=\left(\prod_{i=1}^{p}\left|A_{i}\right|^{-A_{i}}\right)\left(\prod_{j=1}^{q}\left|B_{j}\right|^{B}\right) \\
\mu^{*}=\sum_{j=1}^{q} \beta_{j}-\sum_{i=1}^{p} \alpha_{i}+\left(\frac{p-q}{2}\right) \\
\sigma^{*}=\left(1+A_{1}+\ldots+A_{p}\right)-\left(B_{1}+\ldots+B_{q}\right)=1-\Delta^{*} .
\end{gathered}
$$

Case(I): When contour (L) is a left loop beginning and ending at $-\infty$, then ${ }_{p} \Psi_{q}$ given by (1.4) or (1.6) holds the following convergence conditions

i) when $\Delta^{*}>-1,0<|z|<\infty, z \neq 0$,

ii) when $\Delta^{*}=-1,0<|z|<\delta^{*}$,

iii) when $\Delta^{*}=-1,|z|=\delta^{*}$, and $\Re\left(\mu^{*}\right)>\frac{1}{2}$.

Case(II): When contour (L) is a right loop beginning and ending at $+\infty$, then ${ }_{p} \Psi_{q}$ given by (1.4) or (1.6) holds the following convergence conditions

i) when $\Delta^{*}<-1,0<|z|<\infty, z \neq 0$,

ii) when $\Delta^{*}=-1,|z|>\delta^{*}$,

iii) when $\Delta^{*}=-1,|z|=\delta^{*}$, and $\mathfrak{R}\left(\mu^{*}\right)>\frac{1}{2}$.

Case (III): When contour (L) is starting from $\gamma-i \infty$ and ending at $\gamma+i \infty$, where $\gamma \in \mathrm{R}$, then ${ }_{p} \Psi_{q}$ is also convergent under the following conditions

i) when $\sigma^{*}>0,|\arg (-z)|<\frac{\pi}{2} \sigma^{*}, 0<|z|<\infty, z \neq 0$,

ii) when $\sigma^{*}=0, \arg (-z)=0, \quad 0<|z|<\infty, \quad z \neq 0$ such that $-\gamma \Delta^{*}+\mathfrak{R}\left(\mu^{*}\right)>\frac{1}{2}+\gamma$,

iii) when $\gamma=0, \quad \sigma^{*}=0, \quad \arg (-z)=0, \quad 0<|z|<\infty$, $z \neq 0$ such that, $\mathfrak{R}\left(\mu^{*}\right)>\frac{1}{2}$.

Next we collect some results that we will need in the sequel.

\section{Decomposition identity:}

The idea of separation of a power series into its even and odd terms exhibited by the elementary identity

$$
\sum_{r=0}^{\infty} \Phi(r)=\sum_{r=0}^{\infty} \Phi(2 r)+\sum_{r=0}^{\infty} \Phi(2 r+1)
$$

is atleast as old as the series themselves and concerned power series is absolutely convergent.

Pfaff-Kummer's linear transformation [[5],p.33(eq.19)]:

$$
{ }_{2} F_{1}\left[\begin{array}{c}
a, b ; \\
d ;
\end{array}\right]=(1-z)_{2}^{-a} F_{1}\left[\begin{array}{r}
a, d-b ; \frac{-z}{1-z} \\
d ;,
\end{array}\right.
$$

$\left(a, b \in \mathrm{C} ; \quad d \in \mathrm{C} \backslash \mathrm{Z}_{0}^{-}\right.$and $\left.|\arg (1-z)|<\pi\right)$.

\section{Binomial theorem:}

$$
(1-z)^{-b}={ }_{1} F_{0}\left[\begin{array}{l}
b ; \\
-;
\end{array}\right]=\sum_{r=0}^{\infty} \frac{(b)_{r} z^{r}}{r !}
$$

$\left(|z|<1 ; b \in \mathrm{C} \backslash \mathbf{Z}_{0}^{-}\right)$,

$$
(1-z)^{m}={ }_{1} F_{0}\left[\begin{array}{c}
-m ; z \\
-;
\end{array}\right]=\sum_{r=0}^{m} \frac{(-m)_{r} z^{r}}{r !}, m \in \mathrm{N}_{0} .
$$

In sections 2 and 3, we obtain some reduction formulae for Gauss' hypergeometric function and Fox-Wright hypergeometric function associated with suitable convergence conditions by using binomial theorem, decomposition identity and Pfaff-Kummer's linear transformation. 


\section{Some Reduction Formulae for Gauss' Hypergeometric Function}

The following reduction formulae associated with suitable convergence conditions hold true

$$
\begin{aligned}
& 2 \sqrt{(1+x)}{ }_{2} F_{1}\left[\begin{array}{c}
b, 1-b ; \\
1 / 2 ;
\end{array}\right] \\
& =(\sqrt{(1+x)}+\sqrt{x})^{2 b-1}+(\sqrt{(1+x)}-\sqrt{x})^{2 b-1}, \\
& \left(\left|\frac{x}{1+x}\right|<1 ; 1-2 b \in \mathrm{C} \backslash \mathrm{Z}_{0}^{-}\right) \text {. } \\
& 2(2 b-1) \sqrt{x}{ }_{2} F_{1}\left[\begin{array}{c}
b, 1-b ; \\
3 / 2 ;
\end{array}\right] \\
& =(\sqrt{(1+x)}+\sqrt{x})^{2 b-1}-(\sqrt{(1+x)}-\sqrt{x})^{2 b-1}, \\
& \left(\left|\frac{x}{1+x}\right|<1 ; 1-2 b \in \mathrm{C} \backslash \mathbf{Z}_{0}^{-}\right) \text {. } \\
& 2_{2} F_{1}\left[\begin{array}{c}
-b, b ; \\
1 / 2 ;
\end{array}\right] \\
& =(\sqrt{(1+x)}+\sqrt{x})^{2 b}+(\sqrt{(1+x)}-\sqrt{x})^{2 b}, \\
& \left(\left|\frac{x}{1+x}\right|<1 ;-2 b \in \mathrm{C} \backslash \mathrm{Z}_{0}^{-}\right) \text {. } \\
& 4(b-1) \sqrt{\left(x+x^{2}\right)}{ }_{2} F_{1}\left[\begin{array}{c}
b, 2-b ; \\
3 / 2 ;
\end{array}\right] \\
& =(\sqrt{(1+x)}+\sqrt{x})^{2 b-2}-(\sqrt{(1+x)}-\sqrt{x})^{2 b-2} \text {, } \\
& \left(\left|\frac{x}{1+x}\right|<1 ; 2-2 b \in \mathrm{C} \backslash \mathbf{Z}_{0}^{-}\right) \text {. }
\end{aligned}
$$

Proof of reduction formula (2.1):

Suppose right hand side of equation (2.1) is denoted by $\Omega$. Then by using binomial theorem (1.13), we obtain

$$
\begin{aligned}
& \Omega=(\sqrt{(1+x)}+\sqrt{x})^{2 b-1}+(\sqrt{(1+x)}-\sqrt{x})^{2 b-1} \\
& =(\sqrt{(1+x)})^{2 b-1}\left\{\begin{array}{c}
{ }_{1} F_{0}\left[\begin{array}{c}
1-2 b ; \\
\left.-; \sqrt{\left(\frac{x}{1+x}\right)}\right]
\end{array}\right. \\
+{ }_{1} F_{0}\left[\begin{array}{c}
\left.1-2 b ; \sqrt{\left(\frac{x}{1+x}\right)}\right] \\
-; \sqrt{1+x}
\end{array}\right\}
\end{array}\right\} \\
& =(\sqrt{(1+x}))^{2 b-1} \\
& \times \sum_{r=0}^{\infty}\left\{\frac{(1-2 b)_{r}}{r !}\left(\sqrt{\left(\frac{x}{1+x}\right)}\right)^{r}\left[(-1)^{r}+1\right]\right\} .
\end{aligned}
$$

Now using decomposition identity (1.11) in equation (2.5), we get

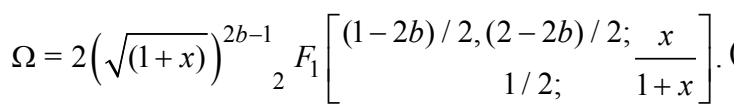

Further using Pfaff-Kummer's linear transformation (1.12) in equation (2.6), and simplifying further, we arrive at left hand side of equation (2.1).

Proof of reduction formula (2.2):

Suppose right hand side of equation (2.2) is denoted by $\Delta$. Then by using binomial theorem (1.13), we get

$$
\begin{aligned}
& \Delta=(\sqrt{(1+x)}+\sqrt{x})^{2 b-1}-(\sqrt{(1+x)}-\sqrt{x})^{2 b-1}
\end{aligned}
$$

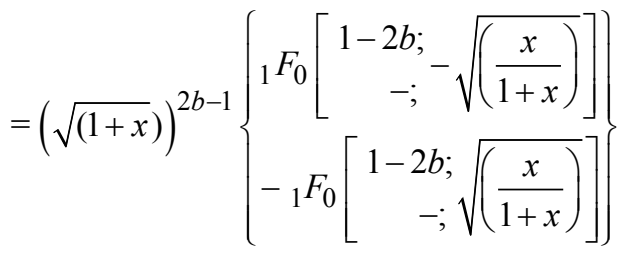

$$
\begin{aligned}
& =(\sqrt{(1+x)})^{2 b-1} \sum_{r=0}^{\infty}\left\{\frac{(1-2 b)_{r}}{r !}\left(\sqrt{\left(\frac{x}{1+x}\right)}\right)^{r}\left[(-1)^{r}-1\right]\right\} \text {. }
\end{aligned}
$$

Now using decomposition identity (1.11) in equation (2.7), we get

$$
\Delta=2 \sqrt{x}(2 b-1)(1+x)^{b-1} F_{1}\left[\begin{array}{r}
-b+1,-b+3 / 2 ; x \\
3 / 2 ; 1+x
\end{array}\right]
$$

On using Pfaff-Kummer's linear transformation (1.12) in equation (2.8), and simplifying further, we have left hand side of equation (2.2).

\section{Proof of reduction formula (2.3):}

Suppose right hand side of equation (2.3) is denoted by $\Lambda$. Then by using binomial theorem (1.13), we have

$$
\begin{aligned}
& \Lambda=(\sqrt{(1+x)}+\sqrt{x})^{2 b}+(\sqrt{(1+x)}-\sqrt{x})^{2 b} \\
& =(\sqrt{(1+x)})^{2 b}\left\{\begin{array}{c}
{ }_{1} F_{0}\left[\begin{array}{c}
\left.-2 b ;-\sqrt{\left(\frac{x}{1+x}\right)}\right] \\
-;
\end{array}\right] \\
+{ }_{1} F_{0}\left[-2 b ; \sqrt{\left(\frac{x}{1+x}\right)}\right]
\end{array}\right\} \\
& =(\sqrt{(1+x)})^{2 b} \sum_{r=0}^{\infty}\left\{\frac{(-2 b)_{r}}{r !}\left(\sqrt{\left(\frac{x}{1+x}\right)}\right)^{r}\left[(-1)^{r}+1\right]\right\} .
\end{aligned}
$$

Now using decomposition identity (1.11) in equation (2.9), we get

$$
\Lambda=2(1+x)_{2}^{b} F_{1}\left[\begin{array}{r}
-b,-b+1 / 2 ; x \\
1 / 2 ; 1+x
\end{array}\right] .
$$

On using Pfaff-Kummer's linear transformation (1.12) in equation (2.10), and simplifying further, we arrive at left hand side of equation (2.3).

Proof of reduction formula (2.4):

Suppose right hand side of equation (2.4) is denoted by $\Xi$. Then by using binomial theorem (1.13), we obtain

$$
\begin{aligned}
& \Xi=(\sqrt{(1+x)}+\sqrt{x})^{2 b-2}-(\sqrt{(1+x)}-\sqrt{x})^{2 b-2} \\
& =(\sqrt{(1+x)})^{2 b-2}\left\{\begin{array}{r}
{ }_{1} F_{0}\left[\begin{array}{c}
2-2 b ; \\
-;
\end{array} ; \sqrt{\left(\frac{x}{1+x}\right)}\right] \\
-{ }_{1} F_{0}\left[\begin{array}{r}
2-2 b ; \\
\left.-; \sqrt{\left(\frac{x}{1+x}\right)}\right]
\end{array}\right\}
\end{array}\right\}
\end{aligned}
$$




$$
=(\sqrt{(1+x)})^{2 b-2} \sum_{r=0}^{\infty}\left\{\begin{array}{l}
\frac{(2-2 b)_{r}}{r !}\left(\sqrt{\left(\frac{x}{1+x}\right)}\right)^{r} \\
\times\left[(-1)^{r}-1\right]
\end{array}\right\} .
$$

Now using decomposition identity (1.11) in equation (2.11), we get

$$
\begin{aligned}
\Xi= & 4 \sqrt{x}(b-1)(1+x)^{b-3 / 2} \\
& \times{ }_{2} F_{1}\left[\begin{array}{r}
-b+2,-b+3 / 2 ; x \\
3 / 2 ; 1+x
\end{array}\right] .
\end{aligned}
$$

Applying Pfaff-Kummer's linear transformation (1.12) in equation (2.12), and simplifying further, we get left hand side of equation (2.4).

\section{Applications of Reduction Formulae in Fox-Wright function}

As an application of formulae (2.1)-(2.4), we obtain the following two formulae for Fox-Wright hypergeometric function associated with convergence conditions

$$
{ }_{1} \Psi_{1}^{*}\left[\begin{array}{c}
(b, 1 / 2) ; z \\
(b,-1 / 2) ;
\end{array}\right]=\frac{2}{\sqrt{\left(z^{2}+4\right)}}\left(\frac{z+\sqrt{\left(z^{2}+4\right)}}{2}\right)^{2 b-1}
$$

and

$$
\begin{gathered}
{ }_{1} \Psi_{1}^{*}\left[\begin{array}{r}
(b, 1 / 2) ; \\
(1+b,-1 / 2) ;
\end{array}\right]=\left(\frac{z+\sqrt{\left(z^{2}+4\right)}}{2}\right)^{2 b}, \\
\left(\left|\frac{z^{2}}{4+z^{2}}\right|<1 ; b \in \mathrm{C} \backslash\left\{0, \pm \frac{1}{2}, \pm 1, \pm \frac{3}{2}, \pm 2, \pm \frac{5}{2}, \pm 3, \ldots\right\}\right) .
\end{gathered}
$$

\section{Proof of reduction formula (3.1):}

Suppose left hand side of equation (3.1) is denoted by $\zeta$. Then by using the definitions of ${ }_{p} \Psi_{q}^{*}(1.4)$ and (1.5), we obtain

$$
\zeta={ }_{1} \Psi_{1}^{*}\left[\begin{array}{c}
(b, 1 / 2) ; \\
(b,-1 / 2) ;
\end{array}\right]=\sum_{n=0}^{\infty} \frac{(b)_{n / 2} z^{n}}{(b)_{-n / 2} n !} .
$$

Now using decomposition identity (1.11) in equation (3.3), we have

$$
\zeta=\sum_{n=0}^{\infty} \frac{(b)_{n} z^{2 n}}{(b)_{-n}(2 n) !}+\sum_{n=0}^{\infty} \frac{(b)_{(2 n+1) / 2} z^{2 n+1}}{(b)_{-(2 n+1) / 2}(2 n+1) !} .
$$

Solving further, we obtain

$$
\begin{aligned}
\zeta= & { }_{2} F_{1}\left[\begin{array}{c}
b, 1-b ; \\
1 / 2 ;
\end{array}\right] \\
& +\frac{z \Gamma(b+1 / 2)}{\Gamma(b-1 / 2)}{ }_{2} F_{1}\left[\begin{array}{c}
b+1 / 2,-b+3 / 2 ;-z^{2} \\
3 / 2 ;
\end{array}\right] .
\end{aligned}
$$

Now applying the reduction formulae (2.1), (2.4), and simplifying further, we get the right hand side of equation (3.1).

Proof of reduction formula (3.2):

Suppose left hand side of equation (3.2) is denoted by $\Theta$. Then by using the definitions of ${ }_{p} \Psi_{q}{ }^{*}$ (1.4) and (1.5), we get

$$
\Theta={ }_{1} \Psi_{1}^{*}\left[\begin{array}{r}
(b, 1 / 2) ; \\
(1+b,-1 / 2) ;
\end{array}\right]=\sum_{n=0}^{\infty} \frac{(b)_{n / 2} z^{n}}{(1+b)_{-n / 2} n !} .
$$

On using decomposition identity (1.11) in equation (3.5), we get

$$
\Theta=\sum_{n=0}^{\infty} \frac{(b)_{n} z^{2 n}}{(1+b)_{-n}(2 n) !}+\sum_{n=0}^{\infty} \frac{(b)_{(2 n+1) / 2} z^{2 n+1}}{(1+b)_{-(2 n+1) / 2}(2 n+1) !} .
$$

Solving further, we get

$$
\begin{aligned}
\Theta= & { }_{2} F_{1}\left[\begin{array}{c}
b,-b ; \\
1 / 2 ;
\end{array}\right] \\
& +\frac{z(b)_{1 / 2}}{(1+b)_{-1 / 2}}{ }_{2} F_{1}\left[\begin{array}{c}
b+1 / 2,-b+1 / 2 ;-z^{2} \\
3 / 2 ;
\end{array}\right] \\
= & { }_{2} F_{1}\left[\begin{array}{c}
b,-b ; \\
1 / 2 ;
\end{array}\right] \\
& +z b_{2} F_{1}\left[\begin{array}{c}
b+1 / 2,-b+1 / 2 ;-z^{2} \\
3 / 2 ;
\end{array}\right] .
\end{aligned}
$$

Now applying the reduction formulae (2.2), (2.3), and solving further, we obtain the right hand side of equation (3.2).

\section{Acknowledgements}

The Authors are thankful to the anonymous referee for his/her valuable remarks and suggestions in the preparation of revised version of this paper.

\section{References}

[1] Boersma J.; On a function which is a special case of Meijer's G-function, Compositio Math., 15 (1962), 34-63.

[2] Braaksma B.L.J.; Asymptotic expansions and analytic continuations for a class of Barnes-integrals, Compositio Math., 15 (1964), 239-341.

[3] Fox C.; The asymptotic expansion of generalized hypergeometric functions, Proc. London Math. Soc., 27(2) (1928), 389-400.

[4] Fox C.; The $G$ and $H$ functions as symmetrical Fourier kernels, Trans. Amer. Math. Soc., 98 (1961), 395-421.

[5] Srivastava H.M. and Manocha H.L.; A Treatise on Generating functions, Halsted Press (Ellis Horwood Ltd., Chichester, U.K.), John Wiley and Sons, New York, Chichester, Brisbane and Toronto, 1984.

[6] Wright E. M.; The asymptotic expansion of the generalized hypergeometric function- I, J. London Math. Soc., 10(4) (1935), 286-293.

[7] Wright E.M.; The asymptotic expansion of the generalized hypergeometric function-II, Proc. London Math. Soc.(2), 46 (1940), 389-408. 